\title{
Factors affecting visual acuity after accelerated crosslinking in patients with progressive keratoconus
}

\author{
Fatores que afetam a acuidade visual após crosslinking acelerado entre pacientes com ceratocone progressivo
}

Ahmet KIrgiz ${ }^{1}$, Kürşat Atalay ${ }^{1}$, Kübra ŞerefoĞlu Çabuk ${ }^{1}$, Havva Kaldirim ${ }^{1}$, Muhittin TaşKapilı $I^{2}$

\begin{abstract}
Purpose: The present study aimed to report the outcomes of patients with progressive keratoconus who were treated via accelerated crosslinking (CXL) 6 months earlier and to determine the factors that promoted improved visual acuity after treatment.

Methods: This retrospective study included 35 eyes of 34 patients with progressive keratoconus who underwent CXL. Topographical measurements were obtained preoperatively and in the first, third, and sixth months postoperatively using a rotating Scheimpflug camera. The uncorrected visual acuity (UCVA), best-corrected visual acuity (BCVA), flat keratometry (K) value (K1), steep $K$ value (K2), average $K$ value (avgK), topographic cylindrical value (Cyl), apical keratoscopy front (AKf) apical keratoscopy back (AKb), symmetry index front (SIf), symmetry index back (SIb), and thinnest point of the cornea (ThkMin) were recorded.

Results: At the 6-month follow-up, the mean UCVA and BCVA values were improved, and the K values remained stable. Statistically significant decreases in AKf $(p=0.04)$ and the thinnest point of the cornea $(p=0.001)$ and a statistically significant increase in AKb $(p=0.01)$ were observed. A correlation analysis revealed that the preoperative BCVA, UCVA, K1, K2, avgK, AKf, and AKb values significantly affected visual acuity at the 6-month follow-up.

Conclusions: Accelerated $C X L$ is an effective treatment for the prevention or even reversal of keratoconus progression. The preoperative $K$ values and apexes of the anterior and posterior cornea were found to affect visual acuity at 6 months after accelerated CXL. Both AKb steepening and AKf flattening appear to be important factors in the stabilization of keratometric values and improvement of visual outcomes.
\end{abstract}

Keywords: Cornea; Keratoconus/therapy; Riboflavin/therapeutic use; Ultraviolet rays; Cross-linking reagents; Visual acuity

\section{RESUMO}

Objetivo: O objetivo do estudo é relatar os resultados do sexto mês após o tratamento de crosslinking acelerado (CXL) em pacientes com ceratocone progressivo e determinar os fatores que afetam a melhora da acuidade após o tratamento.

Métodos: Neste estudo retrospectivo, foram incluidos 35 olhos de 34 pacientes com ceratocone progressivo que se submeteram CXL. Acuidade visual não corrigida (UCVA) e melhor acuidade visual corrigida (BCVA) foram registradas. Medidas topográficas foram obtidas utilizando uma câmara Scheimpflug rotativa no pré-operatório e no 10 , 3o e 6o meses após a cirurgia. Os valores de ceratometria (K) mais plana (K1), K mais curva (K2), médio de K (avgK), astigmatismo topográfico (Cyl), ápice anterior da ceratoscopia (AKf), ápice posterior da ceratoscopia (AKb), índice anterior de simetria (SIf), índice posterior de simetria (SIb) e ponto mais fino da córnea (ThkMin) foram avaliados.

Resultados: A média UCVA e BCVA melhoraram, enquanto valores de K ficaram estáveis $6^{\circ}$ mês. Houve uma diminuição estatisticamente significativa na AKf e um aumento estatisticamente significativo na AKb ( $p=0,04, p=0,01$, respectivamente). O ponto mais fino da córnea diminuiu significativamente $(p=0,001)$. Na análise de correlações, além da UCVA e BCVA pré-operatórias; valores K1, K2, avgK, AKf e AKb pré-operatórios influenciaram significativamente a acuidade visual no 6 mês de acompanhamento. Conclusões: CXL acelerado é uma forma eficaz de tratamento na prevenção ou no mesmo inversão da progressão do ceratocone. A acuidade visual no 60 mês após CXL acelerado foi afetada a partir dos valores de Ke dos ápice anterior e posterior da córnea. Encurvamento do AKb e aplanamento do AKf parecem ser fatores importantes na estabilização dos valores ceratométricos e na melhora da acuidade visual.

Descritores: Córnea; Ceratocone/terapia; Riboflavina/uso terapêutico; Raios ultravioleta; Reagentes para ligações cruzadas; Acuidade visual

\section{INTRODUCTION}

Keratoconus is a bilateral, asymmetric, degenerative disorder of the cornea with an unknown etiology and is characterized by progressive distortion of the anterior corneal surface and apical thinning of the stroma, leading to significant visual morbidity ${ }^{(1)}$. Currently, corneal collagen crosslinking (CXL), which induces covalent binding between individual collagen fibers to increase the stiffness and rigidity of the anterior corneal stroma, is considered the most effective treatment modality for delaying the progression of keratoconus ${ }^{(2-4)}$. CXL treatment relies on a photochemical reaction between riboflavin (vitamin B2) and ultraviolet A (UVA); specifically, riboflavin acts as a photosensitizer to induce crosslinking between collagen fibrils and as a shield to protect underlying tissues from UVA damage ${ }^{(5)}$. Accele- rated CXL delivers a higher irradiation dose to the cornea and reduces the required light exposure time, thus improving patient comfort and decreasing the likelihood of complications ${ }^{(6)}$.

$\mathrm{CXL}$ alters the corneal shape and structure and improves visual acuity, and thus the results of this treatment may be affected by structural changes ${ }^{(7)}$. In particular, CXL is known to reduce the anterior corneal keratometric values; however, elucidation of the postoperative posterior corneal changes requires further investigation ${ }^{(2-4)}$. Although previous studies have evaluated biomechanical and clinical changes in this context, the effects of these changes on visual acuity remain unclear ${ }^{(8)}$. In this study, we aimed to report the 6-month results of accelerated CXL in patients treated for progressive keratoconus and to determine the factors affecting visual acuity after treatment.
Submitted for publication: October 22, 2015

Accepted for publication: February 11, 2016

${ }^{1}$ Department of Opthalmology, Bagcilar Training and Research Hospital, Istanbul, Turkey.

Beyoglu Eye Training and Research Hospital, Istanbul, Turkey.
Funding: No specific financial support was available for this study.

Disclosure of potential conflicts of interest: None of the authors have any potential conflicts of interest to disclose.

Corresponding author: Ahmet Kırgız. Bagcilar Training and Research Hospital, Eye Clinic. Merkez MH, Mimar Sinan CD, 6 - Istanbul - Turkey - E-mail: ahmetk1@yahoo.com

Approved by the following research ethics committee: Bagcilar Training and Research Hospital (\# 213, 13 May 2015). 


\section{METHODS}

This retrospective study was approved by the local ethics committee. The subjects included 35 eyes (19 right, 16 left) of 34 patients (14 female, 20 male; mean age: $24.77 \pm 6.87$ years) with progressive keratoconus who underwent CXL between July 2014 and January 2015 at in Bagcilar Education and Research Hospital, Istanbul, Turkey. Progressive keratoconus was defined as an increase of at least 1.00 diopter (D) in the steepest keratometry (K) measurement or the loss of at least 2 lines in the best corrected distance visual acuity within 1 year. Patients with history of corneal surgery, chemical injury, or delayed epithelial healing, those with a corneal pachymetry less than $400 \mu \mathrm{m}$, and women who were pregnant or lactating during the course of the study were excluded.

All patients underwent accelerated CXL according to the following procedure. Initially, a topical anesthetic agent was administered, and the central 8.0-mm epithelium was removed with a blunt spatula. Riboflavin $(0.1 \%$ in $20 \%$ dextran solution) was then administered topically every 3 minutes for 30 minutes. The cornea was aligned and exposed to UVA (365 nm) for 5 minutes at an irradiance of $18 \mathrm{~mW} / \mathrm{cm}^{2}$ (Peschke Meditrade GmbH, Hünenberg, Switzerland). Isotonic riboflavin administration was continued every minute during UVA exposure. After treatment, the eye surface was washed with $20.0 \mathrm{~mL}$ of a balanced salt solution. Postoperatively, antibiotic and corticosteroid drops were administered and a soft contact lens bandage was placed. This contact lens was removed after the closure of the epithelial defect. Antibiotics and corticosteroid drops were continued 4 times daily for 1 week and 2 weeks, respectively. Patients were examined before surgery and at 1-, 3-, and 6-month intervals after corneal CXL treatment.

Visual acuity was determined using Snellen charts, and scores were converted to logMAR units for analysis. The uncorrected visual acuity (UCVA) and best corrected visual acuity (BCVA) were recorded. The following topographical measurements were obtained preoperatively and at 1, 3, and 6 months postoperatively using a rotating Scheimpflug camera (Sirius, Costruzione Strumenti Oftalmici, Italy) and recorded: flat keratometry (K) value (K1), steep K value (K2), average $K$ value (avgK), topographic cylindrical value (topographic astigmatism) (Cyl), apical keratoscopy front (AKf), apical keratoscopy back (AKb), symmetry index front (SIf), symmetry index back (SIb), and thinnest point of the cornea (minimum corneal thickness; ThkMin). The AKf (maximum keratometric value) was defined as the steepest point of the anterior corneal surface, whereas the AKb was defined as the steepest point of the posterior corneal surface. SIf, the symmetry index of the anterior curvature, was defined as the difference of the mean anterior tangential curvatures of 2 circular zones centered on the vertical axis in the inferior and superior hemispheres. Similarly, $\mathrm{SIb}$, the symmetry index of the posterior curvature, was defined as the difference of the mean posterior tangential curvatures of 2 circular zones centered on the vertical axis in the inferior and superior hemispheres.

\section{Statistical analysis}

SPSS software (version 21; IBM SPSS Statistics, Chicago, IL, USA) was used for the statistical analyses of the results. These results are presented as means \pm standard deviations for continuous variables and as proportions (\%) for categorical variables. Student's $t$-test for paired data was used for most analyses; however, the Pearson correlation test was used for the correlation analysis. A $p$ value of $<0.05$ was considered statistically significant.

\section{RESULTS}

According to a Snellen chart evaluation of UCVA at the 6-month follow-up, UCVA remained stable in 19 (54.3\%) eyes, improved by 1 line in 7 (20.0\%) eyes, improved by 2 lines in 3 (8.6\%) eyes, and improved by $\geq 3$ lines in $2(5.7 \%)$ eyes. However, UCVA decreased by 1 line in 2 (5.7\%) eyes and by 2 lines in 2 (5.7\%) eyes. A similar evaluation of BCVA at the 6-month follow-up, revealed a stable BCVA in 12 (34.3\%) eyes, improvement by 1 line in 13 (37.1\%) eyes, 2 lines in 5 (14.2\%) eyes, and $\geq 3$ lines in 2 (5.7\%) eyes, and a decrease by 1 line in $3(8.6 \%)$ eyes.

The results of the preoperative and 6-month follow-up evaluations are summarized in table 1. The mean UCVA and BCVA values improved, whereas the $K$ values remained stable with topographic astigmatism (Figures 1 and 2). Statistically significant decreases in

Table 1. Preoperative period and 6-month follow-up data

\begin{tabular}{lccc}
\hline & Preoperative period & 6-month follow-up & p \\
\hline UCVA (logMAR) & $0.76 \pm 0.28$ & $0.68 \pm 0.29$ & $\mathbf{0 . 0 2 0}$ \\
BCVA (logMAR) & $0.43 \pm 0.22$ & $0.34 \pm 0.20$ & $\mathbf{0 . 0 1 0}$ \\
K1 (D) & $45.74 \pm 2.01$ & $45.66 \pm 2.15$ & 0.500 \\
K2 (D) & $48.94 \pm 2.40$ & $48.75 \pm 2.50$ & 0.090 \\
Avg K (D) & $47.28 \pm 2.10$ & $47.15 \pm 2.20$ & 0.240 \\
Cyl & $3.20 \pm 1.42$ & $3.10 \pm 1.40$ & 0.080 \\
AKf (D) & $55.76 \pm 3.99$ & $55.25 \pm 4.26$ & $\mathbf{0 . 0 4 0}$ \\
AKb (D) & $81.37 \pm 10.09$ & $84.71 \pm 10.25$ & $\mathbf{0 . 0 1 0}$ \\
SIf & $6.42 \pm 2.87$ & $6.00 \pm 2.82$ & $\mathbf{0 . 0 0 1}$ \\
SIb & $1.63 \pm 0.70$ & $1.67 \pm 0.75$ & 0.390 \\
ThkMin (Mu) & $455.97 \pm 31.46$ & $422.66 \pm 40.11$ & $\mathbf{0 . 0 0 1}$ \\
\hline
\end{tabular}

$\mathrm{UCVA}=$ uncorrected visual acuity; $\mathrm{BCVA}=$ best corrected visual acuity; $\mathrm{K} 1=$ flat keratometry value; $\mathrm{K} 2$ = steep keratometry value; $\mathrm{Avg} \mathrm{K}=$ average keratometry value; $\mathrm{Cyl}=$ topographic cylindrical value; $A K f=$ apical keratoscopy front; $A K b=$ apical keratoscopyback; $S I f=s y m m e t r y$ index front; $\mathrm{Slb}=$ symmetry index back; ThkMin= thinnest point of the cornea; $\mathrm{D}=$ diopters

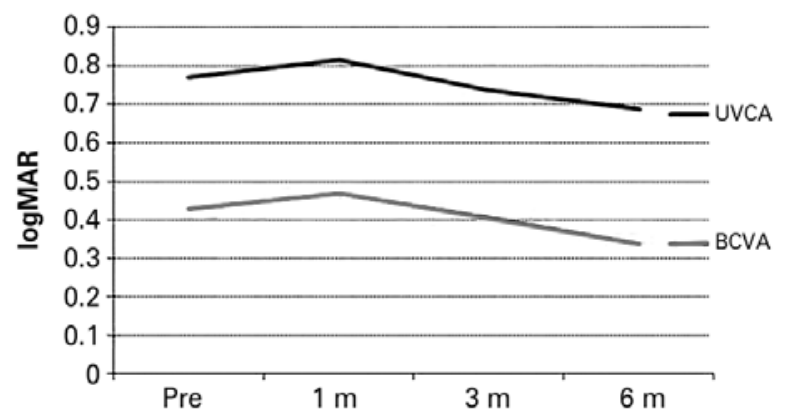

Figure 1. Alterations in the best corrected visual acuity (BCVA) and uncorrected visual acuity (UCVA) values during follow-up.

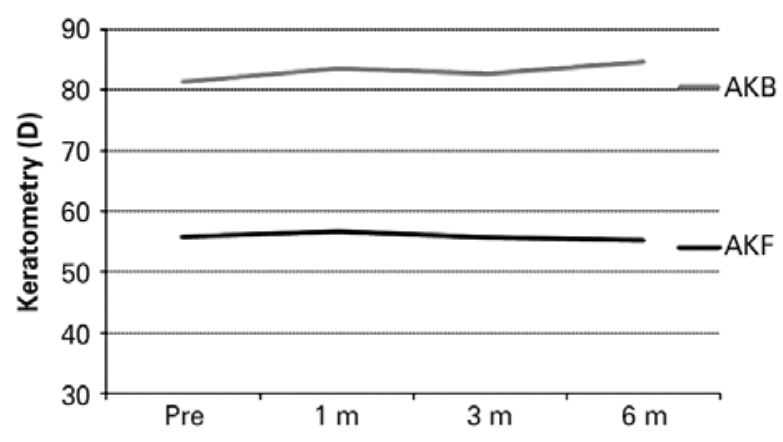

Figure 2. Alterations in the apical keratoscopy front (AKf) and apical keratoscopy back $(\mathrm{AKb})$ values during follow-up. 
AKf and SIf and a significant increase in AKb were observed $(p=0.04$, $p=0.001, p=0.01$, respectively). In addition, the ThkMin exhibited a statistically significant decrease after 6 months ( $p=0.001$; Figure 3 ).

Table 2 summarizes the results of a correlation analysis of the parameters related to visual acuity at the 6-month follow-up. The preoperative BCVA, UCVA, K1, K2, average K, Akf, and Akb values were found to significantly affect the visual acuity at the 6-month follow-up.

\section{DISCUSSION}

In this study, we analyzed the factors affecting visual acuity at a 6-month follow- up evaluation in patients treated with accelerated

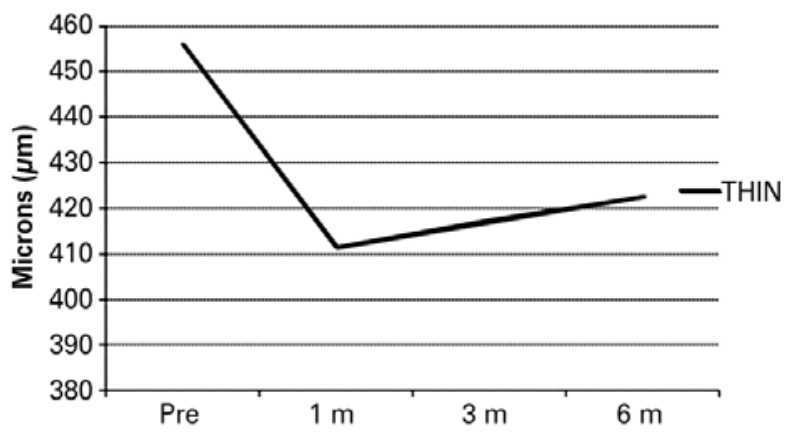

Figure 3. Alterations in the corneal thinnest point during follow-up.

Table 2. Results of the correlation analysis

\begin{tabular}{|c|c|c|c|c|}
\hline & \multicolumn{2}{|c|}{ BCVA $^{a}$} & \multicolumn{2}{|c|}{ UCVA $^{b}$} \\
\hline & $r$ & p & $\mathbf{R}$ & $\mathbf{p}$ \\
\hline Age & -0.050 & 0.760 & 0.13 & 0.460 \\
\hline $\mathrm{BCVA}^{\mathrm{a}}$ & 0.770 & 0.010 & 0.47 & 0.004 \\
\hline UCVA $^{a}$ & 0.460 & 0.005 & 0.76 & 0.010 \\
\hline $\mathrm{K} 1^{\mathrm{a}}$ & 0.530 & 0.001 & 0.37 & 0.020 \\
\hline $\mathrm{K} 2^{\mathrm{a}}$ & 0.440 & 0.008 & 0.56 & 0.010 \\
\hline Avg Ka & 0.500 & 0.002 & 0.49 & 0.003 \\
\hline$\left.C y\right|^{a}$ & 0.001 & 0.990 & 0.41 & 0.010 \\
\hline$A K f^{a}$ & 0.450 & 0.006 & 0.23 & 0.170 \\
\hline$A K b^{a}$ & 0.540 & 0.001 & 0.36 & 0.030 \\
\hline Slba & 0.120 & 0.490 & 0.10 & 0.530 \\
\hline SIfa & -0.020 & 0.880 & 0.03 & 0.840 \\
\hline ThkMin ${ }^{a}$ & -0.190 & 0.260 & -0.12 & 0.490 \\
\hline $\mathrm{K} 1^{\mathrm{b}}$ & 0.520 & 0.001 & 0.39 & 0.020 \\
\hline$K 2^{\mathrm{b}}$ & 0.490 & 0.002 & 0.59 & 0.010 \\
\hline Avg $K^{b}$ & 0.530 & 0.001 & 0.52 & 0.001 \\
\hline$\left.C y\right|^{b}$ & 0.080 & 0.640 & 0.44 & 0.008 \\
\hline$A K f^{\circ}$ & 0.510 & 0.001 & 0.39 & 0.040 \\
\hline$A K b^{b}$ & 0.640 & 0.010 & 0.35 & 0.040 \\
\hline$S \mid f^{b}$ & -0.080 & 0.640 & -0.03 & 0.860 \\
\hline$S I b^{b}$ & 0.090 & 0.600 & 0.04 & 0.840 \\
\hline ThkMin ${ }^{b}$ & -0.220 & 0.200 & 0.01 & 0.960 \\
\hline
\end{tabular}

$\mathrm{UCVA}=$ uncorrected visual acuity; $\mathrm{BCVA}=$ best corrected visual acuity; $\mathrm{K} 1=$ flat keratometry value; $\mathrm{K} 2$ = steep keratometry value; $\mathrm{Avg} \mathrm{K}=$ average keratometry value; $\mathrm{Cyl}=$ topographic cylindrical value; $\mathrm{AKf}=$ apical keratoscopy front; $\mathrm{AKb}=$ apical keratoscopy back; $\mathrm{SIf}=$ symmetry index front; $\mathrm{SIb}=$ symmetry index back; ThkMin= thinnest point of the cornea; $r=$ correlation coefficient. ${ }^{a}=$ for preoperative; ${ }^{b}=$ for 6 -month follow-up.
CXL for progressive keratoconus and determined that the preoperative $\mathrm{K} 1, \mathrm{~K} 2$, average $\mathrm{K}, \mathrm{Akf}$, and $\mathrm{Akb}$ values had significant effects on visual acuity at this follow-up time point. Notably, visual acuity improved significantly after treatment as the AKf decreased and AKb increased. Although we did not observe statistically significant differences between the pre-and post-operative K1, K2, and average K values, we found that all $\mathrm{K}$ values, including Akf, affected visual acuity. Notably, we observed negative correlations between $\mathrm{K}$ values and visual acuity; in other words, lower preoperative $\mathrm{K}$ values were associated with better visual acuity at the 6-month follow-up. Although the corneal thickness decreased after treatment, we did not observe a correlation between this parameter and visual acuity. Furthermore, we observed a statistically significant increase in postoperative AKb values, as well as positive correlations between visual acuity and AKb values during both the preoperative and postoperative periods.

Corneal collagen CXL, which increases the biomechanical stability of the cornea, is the preferred treatment for the management of progressive keratoconus ${ }^{(9)}$. McAnea and O'Keefe described the visual, refractive, and topographic outcomes following $C X L$ in pediatric patients with keratoconus and reported improvements in BCVA and stable Kmax, Kmin, and Kmean values at 1 year ${ }^{(10)}$. Sedaghat et al. also reported statistically significant improvements in BCVA and UCVA during a 1-year follow-up after CXL, along with significant decreases in the mean average keratometry values and apex corneal thickness ${ }^{(11)}$. Berger et al. further reported stabilization of the average keratometry values along with BCVA values in a 12-month follow-up after CXL; similar to our findings, these results also supported the preventive effects of $C X L$ against keratoconus progression ${ }^{(12)}$.

Accelerated CXL represents a reformation of standard $C X L$ and is characterized by significantly reduced treatment times, patient discomfort, and complication rates. Recently, Hashemi et al. compared the 6-month results of accelerated and standard collagen CXL treatments for progressive keratoconus and determined that the mean changes in uncorrected and corrected visual acuities, as well as the mean decreases in the maximum $\mathrm{K}$ and mean $\mathrm{K}$ values, did not differ statistically significantly between the groups ${ }^{(13)}$. Elbaz et al. retrospectively studied the efficacy of accelerated CXL in 16 keratoconus-affected eyes and reported the effectiveness of this procedure for the stabilization of topographic parameters, including Ksteep, Kflat, average K, corneal astigmatism, and maximal curvature reading at the corneal apex, after 12 months of follow-up ${ }^{(14)}$. Mita et al. also evaluated the effectiveness of accelerated CXL in 39 eyes and reported significantly improved UCVA and Kmax values at 6 months after treatment $\mathrm{t}^{(15)}$.

The maximum keratometry value (Kmax or AKf) has been considered a key topographic indicator of CXL success, and has been reported as stable or decreased after CXL in many studies ${ }^{(3,15-17)}$. Similarly, we also observed significantly decreased AKf values after treatment. In contrast, AKb, which represents the steepest point of the posterior cornea, increased significantly after treatment. Additionally, we observed a positive correlation between the visual acuity and AKb values during both the preoperative period and the 6-month follow-up. In our opinion, this increase in AKb might be associated with reduced corneal thickness and/or flattening of the anterior cornea, and might help to stabilize K values. Notably, these increased AKb values were found to associate with increased visual acuity. To the best of our knowledge, previous studies have not investigated an association of the steepest point of the posterior cornea with visual acuity in the context of CXL, thus warranting further evaluation of this phenomenon in larger studies.

We additionally determined a significant decrease in the ThkMin at 6 months after CXL. Similarly, Derakhshan et al. and Vinciguerra et al. reported a significant decrease in the apex corneal thickness during a 1-year follow-up after CXL but did not report changes between the 6 - and 12-month time points ${ }^{(16,18)}$. However, Greenstein et al. also reported corneal thinning up to 3 months after $C X L$, after 
which this parameter returned to the baseline value after 3-6 months and remained similar to the preoperative value at the 1-year followup $^{(19)}$. Moreover, McAnea and O'Keefe defined a significant reduction in the baseline mean thinnest corneal area at 6 months after $C X L$, followed by a recovery at 1 year ${ }^{(10)}$. In our study, the lowest value for the thinnest corneal point was measured at 1 month after $C X L$; this value increased slightly until the 6-month time point but did not reach preoperative levels. We did not determine any effects of the ThkMin on visual acuity.

Currently, the available information regarding factors affecting the outcome of accelerated CXL is limited. Notably, Toprak et al. reported that in patients with progressive keratoconus, age, baseline visual acuity, and baseline thinnest pachymetry affected the success of CXL treatment ${ }^{(20)}$. In contrast, we did not determine any effects of age or the ThkMin on visual acuity. Recently, De Angelis et al. reported a significant improvement in the 1-year postoperative BCVA, but no significant change in the 1-year postoperative Kmax, and identified a low preoperative BCVA, high refractive astigmatism, and advanced keratoconus as factors predictive of BCVA improvement ${ }^{(21)}$. In contrast, we determined that astigmatism had some significant effects on UCVA but not on BCVA, at a 6-month follow-up. In addition, we also identified a positive correlation between preoperative and postoperative visual acuity but observed that preoperative $\mathrm{K}$ values had a negative effect on visual acuity. In our study, eyes with better preoperative visual acuity and less severe keratoconus (lower $\mathrm{K}$ values) had better visual acuities at 6 months after CXL.

We note that the major limitations of this study are the small sample size and short duration of follow-up. Larger studies with longer follow-up periods will be required to define the factors affecting the outcomes of accelerated CXL.

In conclusion, accelerated CXL is an effective treatment for the prevention or even reversal of keratoconus progression. The $\mathrm{K}$ values and the steepest points of the anterior and posterior cornea were found to affect the visual acuity at 6 months after accelerated CXL. Posterior corneal steepening appears to be as important as anterior corneal flattening for stabilizing the keratometric values and achieving a better postoperative visual outcome.

\section{REFERENCES}

1. Jeyabalan N, Shetty R, Ghosh A, Anandula VR, Ghosh AS, Kumaramanickavel G. Genetic and genomic perspective to understand the molecular pathogenesis of keratoconus. Indian J Ophthalmol. 2013;61(8):384-8.

2. Wollensak G. Crosslinking treatment of progressive keratoconus: New Hope. Curr Opin Opthalmol. 2006;17(4):356-60.
3. Wollensak G, Spoerl E, SeilerT. Riboflavin/ultraviolet-A-induced collagen cross-linking for the treatment of keratoconus. Am J Ophthalmol. 2003;135(5):620-7.

4. Ghanem RC, Santhiago MR, Berti T. Topographic, corneal wavefront, and refractive outcomes 2 years after collagen cross-linking for progressive keratoconus. Cornea. 2014;33(1):43-8.

5. Iseli HP, Thiel MA, Hafezi F, Kampmeier J, Seiler T. Ultraviolet A/riboflavin cornea cross-linking for infectious keratitis associated with corneal melts. Cornea. 2008; 27(5):590-4.

6. Koller T, Mrochen M, Seiler T. Complication and failure rates after corneal cross-linking J Cataract Refract Surg. 2009;35(8):1358-62.

7. Greenstein SA, Fry KL, Hersh PS. Corneal topography indices after corneal collagen crosslinking for keratoconus and corneal ectasia: One-year results. J Cataract Refract Surg. 2011;37(7):1282-90.

8. Goldich Y, Marcovich AL, Barkana Y, Mandel Y, Hirsh A, Morad Y, Avni I, et al. Clinica and corneal biomechanical changes after collagen cross-linking with riboflavin and UV irradiation in patients with progressive keratoconus: results after 2 years of follow-up. Cornea. 2012;31(6):609-14.

9. Kanellopoulos AJ. Collagen cross-linking in early keratoconus with riboflavin in a femtosecond laser-created pocket: initial clinical results. J Refract Surg. 2009;25(11):1034-7.

10. McAnena L, O'Keefe M. Corneal collagen crosslinking in children with keratoconus. J AAPOS. 2015;19(3):228-32.

11. Sedaghat M, Bagheri M, Ghavami S, Bamdad S. Changes in corneal topography and biomechanical properties after collagen cross linking forkeratoconus: 1-year results. Middle East Afr J Ophthalmol. 2015;22(2):212-9.

12. Berger Y, Ezra-Nimni O, Skaat A, Fogel M, Grinbaum A, Barequet I. Corneal collagen cross-linking novel technique for prevention of keratoconus progression: results after one-year at the Sheba Medical Center. Harefuah. 2015;154(2):118-21.

13. Hashemi H, Fotouhi A, Miraftab M, Bahrmandy H, Seyedian MA, Amanzadeh K, et al. Short-term comparison of accelerated and standard methods of corneal collagen crosslinking. J Cataract Refract Surg. 2015;41(3):533-40.

14. Elbaz U, Shen C, Lichtinger A, Zauberman NA, Goldich Y, Chan CC, et al. Accelerated $\left(9-\mathrm{mW} / \mathrm{cm}^{2}\right)$ corneal collagen crosslinking for keratoconus-A 1-year follow-up. Cornea. 2014;33(8):769-73.

15. Mita M, Waring GO, Tomita M. High-irradiance accelerated collagen crosslinking for the treatment of keratoconus: six-month results. J Cataract Refract Surg. 2014; 40(6):1032-40.

16. Derakhshan A, Shandiz JH, Ahadi M, Daneshvar R, Esmaily H. Short-term outcomes of collagen crosslinking for early keratoconus. J Ophthalmic Vis Res. 2011;6(3):155-9.

17. Raiskup F, Theuring A, Pillunat LE, Spoerl E. Corneal collagen crosslinking with riboflavin and ultraviolet-A light in progressive keratoconus: ten-year results. J Cataract Refract Surg. 2015;41(1):41-6.

18. Vinciguerra $P$, Albè E, Trazza $S$, Rosetta $P$, Vinciguerra $R$, Seiler $T$, et al. Refractive, topographic, tomographic, and aberrometric analysis of keratoconic eyes undergoing corneal cross-linking. Ophthalmology. 2009;116(3):369-78.

19. Greenstein SA, Shah VP, Fry KL, Hersh PS. Corneal thickness changes after cornea collagen crosslinking for keratoconus and corneal ectasia: One-year results. J Cataract Refract Surg. 2011;37(4):691-700.

20. Toprak I, Yaylalı V, Yildirim C. Factors affecting outcomes of corneal collagen crosslinking treatment. Eye (Lond). 2014;28(1):41-6.

21. De Angelis F, Rateau J, Destrieux C, Patat F, Pisella PJ. [Predictive factors for visual outcome after corneal collagen crosslinking treatment in progressive keratoconus: One-year refractive and topographic results]. J Fr Ophtalmol. 2015;38(7):595-606. French. 